\title{
Sensor óptico no auxílio à recomendação de adubação nitrogenada em cana-de-açúcar
}

\author{
Lucas Rios do Amaral(1) e José Paulo Molin(1) \\ (1)Universidade de São Paulo, Escola Superior de Agricultura Luiz de Queiroz, Departamento de Engenharia de Biossistemas, Avenida Pádua \\ Dias, no 11, Caixa Postal 09, CEP 13418-900 Piracicaba, SP. E-mail: lucasamaral@agronomo.eng.br, jpmolin@usp.br
}

\begin{abstract}
Resumo - O objetivo deste trabalho foi avaliar o potencial de um sensor óptico ativo terrestre como auxiliar na recomendação da aplicação de nitrogênio em taxa variável, na cultura da cana-de-açúcar. Foram instalados experimentos em delineamento de blocos ao acaso, com uso de diferentes doses de $\mathrm{N}(0,50,100,150$ e $\left.200 \mathrm{~kg} \mathrm{ha}^{-1}\right)$. A resposta da cana-de-açúcar ao $\mathrm{N}$ foi avaliada por diferentes métodos - sensor óptico, clorofilômetro e teor foliar de $\mathrm{N}-$, quando a altura média dos colmos atingiu 0,2, 0,4 e 0,6 m. Observou-se baixa correlação entre o teor foliar de $\mathrm{N}$ e a quantidade de clorofila nas folhas mensuradas por clorofilômetro. Portanto, essas características foram insuficientes para avaliar a eficiência do sensor óptico, uma vez que os valores mensurados se elevaram conforme o aumento da dose de $\mathrm{N}$. A estratégia de recomendação com base na resposta da cultura, estimada pelo sensor óptico em faixa de cana-de-açúcar que recebeu a dose adequada de $\mathrm{N}$, mostrou-se mais condizente com a produtividade obtida. O sensor óptico é ferramenta útil para auxiliar na recomendação de $\mathrm{N}$ para a cultura da cana-de-açúcar, ao se considerar a variabilidade espacial da sua demanda.
\end{abstract}

Termos para indexação: Saccharum, nitrogênio, agricultura de precisão, sensoriamento remoto.

\section{Optical sensor to support nitrogen fertilization recommendation for sugarcane crops}

\begin{abstract}
The objective of this work was to evaluate the potential of a terrestrial active optical sensor to support nitrogen fertilization recommendation, in variable rates, for sugarcane crops. Experiments in a randomized complete block design were carried out with different $\mathrm{N}$ rates $\left(0,50,100,150\right.$ and $\left.200 \mathrm{~kg} \mathrm{ha}^{-1}\right)$. The response of sugarcane to $\mathrm{N}$ was evaluated by different methods - optical sensor, chlorophyll meter, and leaf $\mathrm{N}$ content -, when the average height of stalks was $0.2,0.4$ and $0.6 \mathrm{~m}$. A low correlation was observed between leaf $\mathrm{N}$ content and the amount of chlorophyll measured by a portable chlorophyll meter. Therefore, these two methods were insufficient to evaluate the efficiency of the optical sensor, since the measured values increased with the amount of $\mathrm{N}$ applied. The strategy of fertilization recommendation based on crop response, estimated by the optical sensor in a sugarcane strip that received the appropriate $\mathrm{N}$ rate, was more consistent with the obtained yield. The optical sensor is a useful tool to recommend $\mathrm{N}$ for sugarcane crops, taking into account the spatial variability of $\mathrm{N}$ demand.
\end{abstract}

Index terms: Saccharum, nitrogen, precision agriculture, remote sensing.

\section{Introdução}

Em razão de suas características de clima e solo, o Brasil é o país com melhores condições para gerar e produzir fontes energéticas alternativas ao petróleo, como o álcool derivado da cana-de-açúcar (etanol), que figura como alternativa que mais atende às exigências das economias mundiais, por ser renovável e poluir menos (Segato et al., 2006). A aplicação de processos mais eficientes, que incrementem a produtividade e a redução dos custos de produção, principalmente a partir do menor uso de insumos, são fundamentais para a atuação do setor sucroalcooleiro em mercados cada vez mais competitivos.

O nitrogênio é um dos insumos que desperta maior atenção dos pesquisadores, ao se considerar a resposta variável das culturas, em razão da sua dinâmica complexa no solo. Cantarella et al. (2007) verificaram que, em cana-de-açúcar, a eficiência de uso do $\mathrm{N}$, fornecido por fertilizantes e mensurado por sua recuperação no sistema, é menor que $40 \%$, valor inferior ao da maioria das culturas, entre 50 e $70 \%$.

De acordo com Solari (2006), são três as principais causas para a baixa eficiência de uso do $\mathrm{N}$ : falta de

Pesq. agropec. bras., Brasília, v.46, n.12, p.1633-1642, dez. 2011 
sincronia entre a demanda de $\mathrm{N}$ pela cultura e o seu fornecimento; aplicações uniformes de $\mathrm{N}$ sem considerar a variabilidade espacial existente dentro de cada talhão e o potencial de resposta da cultura; e a forma como a recomendação do $\mathrm{N}$ é feita, tendo como base uma estimativa de produtividade que, na maioria das vezes, não é atingida. Essa eficiência poderia ser incrementada com a utilização de métodos que estimem a resposta da cultura ao $\mathrm{N}$ em determinada situação edafoclimática durante a safra, o que possibilitaria a aplicação de fertilizantes nitrogenados em taxa variável.

O uso de sensores ópticos ativos terrestres é uma das alternativas à recomendação tradicional de N. Essa estratégia parte do princípio de que as propriedades espectrais das folhas das plantas são afetadas pela deficiência de N (Blackmer et al., 1996), e que se caracterizam por utilizar fonte de luz própria e captar a reflectância de determinados comprimentos de ondas pelo dossel das plantas. O uso desses sensores vem sendo avaliado nas culturas de milho (Blackmer et al., 1996; Teal et al., 2006; Solari et al., 2008), trigo (Lukina et al., 2001; Raun et al., 2002; Povh et al., 2008; Grohs et al., 2009), algodão (Sui et al., 2005; Motomiya et al., 2009) e cevada (Povh et al., 2008; Grohs et al., 2009), entre outras.

No entanto, são escassos os estudos disponíveis para a cana-de-açúcar. Inamasu et al. (2006) e Frasson (2007), ao avaliar a capacidade de diferentes sensores ópticos em identificar as doses de $\mathrm{N}$ aplicadas, concluíram que esses equipamentos são úteis para essa finalidade.

O objetivo deste trabalho foi avaliar a potencialidade de um sensor óptico ativo terrestre como auxiliar na recomendação de $\mathrm{N}$, em taxa variável, na cultura da cana-de-açúcar.

\section{Material e Métodos}

Os experimentos foram conduzidos em área da Usina São Martinho, localizada na região de Ribeirão Preto, SP (21ํ1'34"S e 4803'56"W). Foram selecionados dois talhões de cana-de-açúcar, classificados conforme Prado (2005), como ambiente de produção A (Latossolo Vermelho eutrófico). Os talhões, um em segundo corte (área 1) e o outro em quarto corte (área 2), foram cultivados com a variedade RB85-5453 e colhidos mecanicamente em junho de 2009. Os dados de temperatura e precipitação durante a condução do experimento foram coletados pela estação meteorológica da usina, cerca de $10 \mathrm{~km}$ das áreas experimentais (Figura 1).

$\mathrm{O}$ experimento foi instalado em cada uma das áreas, tendo como tratamentos quatro doses de $\mathrm{N}(50,100$, 150 e $200 \mathrm{~kg} \mathrm{ha}^{-1}$ ) e uma testemunha sem aplicação de $\mathrm{N}$. As doses de $\mathrm{N}$ foram aplicadas manualmente logo após o corte, na forma de nitrato de amônio, em delineamento de blocos ao acaso, com quatro repetições. Cada parcela era constituída de seis fileiras de cana-de-açúcar, espaçadas em $1,5 \mathrm{~m}$, com $15 \mathrm{~m}$ de comprimento, tendo-se utilizado como área útil os $10 \mathrm{~m}$ centrais das quatro fileiras internas. Realizou-se amostragem de solo na profundidade de $0,0-0,2 \mathrm{~m}$, com dez subamostras, para determinação da fertilidade do solo (Tabela 1) (Raij et al., 2001).

As avaliações da reflectância da cultura foram realizadas quando a altura média de colmos na brotação atingiu $0,2,0,4$ e $0,6 \mathrm{~m}$, aproximadamente aos 60, 120 e 145 dias após o corte, respectivamente.

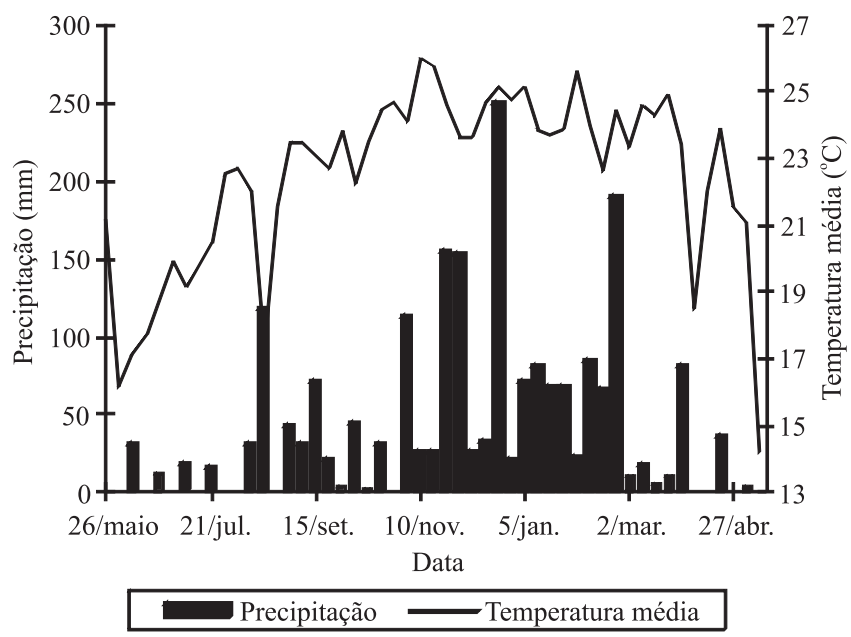

Figura 1. Temperatura média do ar e precipitação na região de Ribeirão Preto, SP, durante o período de condução dos experimentos.

Tabela 1. Atributos químicos do solo na camada de $0,0-0,2 \mathrm{~m}$ de profundidade, nas duas áreas experimentais ${ }^{(1)}$.

\begin{tabular}{ccccccccc}
\hline Área & $\begin{array}{c}\mathrm{MO} \\
\left(\mathrm{g} \mathrm{dm}^{-3}\right)\end{array}$ & $\begin{array}{c}\mathrm{P}_{\text {res }} \\
\left(\mathrm{mg} \mathrm{dm}^{-3}\right)\end{array}$ & $\begin{array}{c}\mathrm{K} \\
-----\end{array}$ & $\begin{array}{c}\mathrm{Ca} \\
\left(\mathrm{mmol}_{\mathrm{c}} \mathrm{dm}^{-3}\right)\end{array}$ & ------ & -------- & $\mathrm{CTC})$ & -------- \\
\hline 1 & 35,6 & 6,0 & 1,4 & 41,9 & 15,8 & 89,9 & 0,17 & 65,71 \\
2 & 34,8 & 25,9 & 4,9 & 36,2 & 9,3 & 102,6 & 0,59 & 49,11 \\
\hline
\end{tabular}

${ }^{(1)} \mathrm{MO}$, matéria orgânica; $\mathrm{P}_{\mathrm{res}}$, fósforo disponível obtido por resina trocadora de íons; CTC, capacidade de troca catiônica; m, saturação por alumínio; V, saturação por bases. 
Utilizou-se o sensor óptico ativo terrestre CropCircle, modelo ACS-210 (Holland Scientific, Inc., Lincoln, NE, EUA), que trabalha com dois comprimentos de onda, na região do visível (âmbar, $590 \pm 5,5 \mathrm{~nm})$ e do infravermelho próximo (IVP, $880 \pm 10 \mathrm{~nm}$ ), com frequência de emissão aproximada de $40.000 \mathrm{~Hz}$, em que a reflectância do alvo é captada por dois fotodetectores (400 a $680 \mathrm{~nm}$ e 800 a $1.100 \mathrm{~nm}$, respectivamente), o que elimina a interferência da luminosidade do ambiente (Solari, 2006). O sensor ficou a uma distância de 0,80 a $1,20 \mathrm{~m}$ do dossel da cultura (recomendações do fabricante), e os dados de reflectância foram coletados dinamicamente, apenas de uma fileira de plantas por vez, com frequência de coleta de até dez dados por segundo. A média dos valores de reflectância mensurados na área útil representou o valor de cada parcela.

A partir dos valores das duas bandas separadamente, foi possível calcular o índice de vegetação por diferença normalizada (NDVI) e o índice de clorofila $\left(\mathrm{CI}_{\text {âmbar }}\right)$ através das equações (1) e (2) respectivamente:

$$
\begin{aligned}
& \mathrm{NDVI}=\left(\mathrm{IVP}_{880}-\text { visível }_{590}\right) /\left(\mathrm{IVP}_{880}+\text { visível }_{590}\right) \\
& \mathrm{CI}_{\text {âmbar }}=\left(\mathrm{IVP}_{880} / \text { visível }_{590}\right)-1
\end{aligned}
$$

O primeiro é largamente utilizado na literatura para a estimativa da nutrição por N (Lukina et al., 2001; Raun et al., 2002; Solari, 2006; Eitel et al., 2008), enquanto o segundo vem sendo indicado por ser mais sensível à variação de clorofila nas folhas, em comparação ao NDVI, principalmente em culturas que apresentam moderada a alta quantidade de biomassa (Gitelson et al., 2003; Solari et al., 2008).

Concomitantemente às avaliações com o sensor óptico, procedeu-se à estimativa do teor foliar de clorofila, a partir do medidor portátil de clorofila SPAD-502 (Konica Minolta Sensing, Inc., Sakai, Osaka, Japão), tendo-se realizado 30 mensurações em 30 plantas por parcela, conforme Raij \& Cantarella (1997), no centro do limbo foliar da folha +1 (primeira folha com aurícula visível). A média foi considerada como o valor de cada parcela. Essas mesmas folhas tiveram sua nervura central excluída e foram enviadas ao Laboratório de Nutrição de Plantas da Escola Superior de Agricultura Luiz de Queiroz, da Universidade de São Paulo, para determinação do teor total de $\mathrm{N}$ foliar (Bataglia et al., 1983).

Um dia antes da colheita, realizou-se a coleta de dez colmos na área útil das parcelas, para determinação, no laboratório da própria usina, de: teores de sólidos solúveis ( ${ }^{\circ}$ Brix) em percentagem no caldo da cana, pol (sacarose) em percentagem no caldo da cana, percentagem de fibra da cana e açúcar teórico recuperável (ATR). A colheita total da área útil de cada parcela foi realizada com pesagem individualizada, a partir de balança embarcada em caminhão.

Os resultados foram submetidos a testes de correlação pelo teste te à análise de variância, e, quando significativos, à análise de regressão. A produtividade final de colmos também foi analisada pelo teste $\mathrm{F}$, por meio de contrastes ortogonais. As análises estatísticas foram realizadas com uso do Sisvar (Ferreira, 2008).

Simultaneamente ao experimento em parcelas, realizou-se a coleta de dados com o sensor óptico (NDVI e $\mathrm{CI}_{\text {âmbar }}$ ), em área total dos talhões experimentais, que apresentavam área de 9 e 11 ha, nas áreas 1 e 2, respectivamente. Essa avaliação teve por objetivo analisar metodologias de recomendação de N, com base no sensor óptico. Para tanto, conectou-se o sensor óptico a um receptor GPS AG 132 (Trimble, Sunnyvale, CA, EUA), com correção por algoritmo interno e precisão da ordem de $0,3 \mathrm{~m}$ em passadas paralelas. Realizou-se a mensuração a cada três fileiras de cana, com frequência de coleta de um dado por segundo, a partir do acoplamento do sensor óptico e do receptor GPS a um veículo adubador Uniport 3000 NPK (Jacto Máquinas Agrícolas S.A., Pompéia, SP).

Foram utilizados dois métodos para converter os valores de NDVI e $\mathrm{CI}_{\text {âmbar }}$ em recomendações de N, em taxa variável. O primeiro, adaptado de Lukina et al. (2001), estima a quantidade de $\mathrm{N}$ a ser aplicada, com base na curva de dose/resposta da cultura ao $\mathrm{N}$ obtida nos experimentos em parcelas, a partir de mensurações feitas com o sensor óptico (Met-CDR), em que um valor qualquer do índice de vegetação (IV) indica uma quantidade teórica de $\mathrm{N}$ fornecido pelo solo ( $\mathrm{N}$ absorvido). Recomenda-se a diferença entre a dose máxima de $\mathrm{N}$, para cada área experimental, e a quantidade de $\mathrm{N}$ absorvida.

O segundo método foi proposto por Raun et al. (2002), para a cultura do trigo, e testado e adaptado, no Brasil, por Povh (2007). Suas variáveis determinantes são: a estimativa da produtividade, a partir de mensurações com o sensor óptico; e a resposta da cultura ao $\mathrm{N}$ durante o seu desenvolvimento, o que requer a instalação de uma faixa da cultura rica em $\mathrm{N}$, que proporcione o máximo desenvolvimento da 
cana-de-açúcar (Met-FRN). Assim, a produtividade é estimada com base nos valores mensurados com o sensor óptico, em campo (YP0). Em seguida, o índice de resposta (RI) é calculado ao se dividir os valores dos índices de vegetação médios, mensurados na faixa rica em N $\left(200 \mathrm{~kg} \mathrm{ha}^{-1}\right)$, pelos valores do índice de vegetação, em cada ponto coletado em área total. Multiplicou-se o YP0 pelo RI, para obter o potencial produtivo com a aplicação de N (YPN) em cada ponto.

Após a determinação do potencial produtivo das plantas, com ou sem aplicação de N, calculou-se a dose de $\mathrm{N}$ a ser aplicada (Equação 3), de acordo com cada índice de vegetação, tendo-se aplicado $1 \mathrm{~kg} \mathrm{ha}^{-1}$ de $\mathrm{N}$ para cada tonelada de colmo esperada (Vitti \& Mazza, 2002), com aproveitamento da adubação de 40\% (Cantarella et al., 2007).

Dose de N $\left(\mathrm{kg} \mathrm{ha}^{-1}\right)=[(\mathrm{YPN}-\mathrm{YP} 0) \times 1] / 0,4$

O tratamento dos dados obtidos pelo sensor óptico (NDVI e $\mathrm{CI}_{\text {âmbar }}$ ) foi realizado em planilha eletrônica do programa Excel (Microsoft Office), tendo-se calculado as quantidades de $\mathrm{N}$ necessárias pelas duas metodologias. Os dados foram analisados por estatística descritiva.

\section{Resultados e Discussão}

A produtividade de colmos foi altamente variável dentro da área experimental, o que dificultou a obtenção de resposta significativa às doses de $\mathrm{N}$ (Figura 2). Observou-se diferença significativa apenas entre as médias dos tratamentos com e sem adubação de $\mathrm{N}$ (testemunha) (Tabela 2). As doses de $\mathrm{N}$ também não interferiram nos parâmetros de qualidade da cana (Tabela 3), em concordância com Vitti et al. (2007). De acordo com Cantarella et al. (2007), estudos com $\mathrm{N}$ feitos em campo, quando analisados separadamente, muitas vezes não atingem significância estatística, em virtude do grande erro experimental, mas quando os dados são analisados em conjuntos de experimentos, observa-se importante resposta a esse elemento.

Quanto aos teores foliares de N, não houve variação na área 1 , enquanto na área 2 , verificou-se resposta linear na segunda avaliação, mas não na terceira (Tabela 4). Isso pode ser atribuído ao efeito diluição, já que a concentração dos nutrientes é diluída com o maior crescimento da planta, nos tratamentos que recebem altas doses de fertilizantes nitrogenados
(Jarrell \& Beverly, 1981). Esses resultados estão de acordo com Raij \& Cantarella (1997), que afirmam que, para cana-de-açúcar, a técnica de determinação nutricional da cultura, por meio de análises foliares, ainda é um desafio.

Em relação aos teores de clorofila das folhas, representados pelo índice SPAD mensurado pelo clorofilômetro, observou-se ausência de resposta na primeiraavaliação, em virtude dolento desenvolvimento das plantas (Tabela 4). A partir da segunda avaliação, pôde-se observar diferença na Área 1, o que mostra a maior eficiência do equipamento em indicar a nutrição da planta por $\mathrm{N}$, em comparação à análise foliar em cana-de-açúcar. Entretanto, na Área 2, os resultados foram semelhantes aos observados para o teor foliar de $\mathrm{N}$, provavelmente por esse mesmo motivo.

O teor de clorofila pode ser utilizado para estimar indiretamente o teor de N (Argenta et al., 2001; Hurtado et al., 2009; Rambo et al., 2011). No entanto, ao comparar os dois métodos avaliados, verificou-se

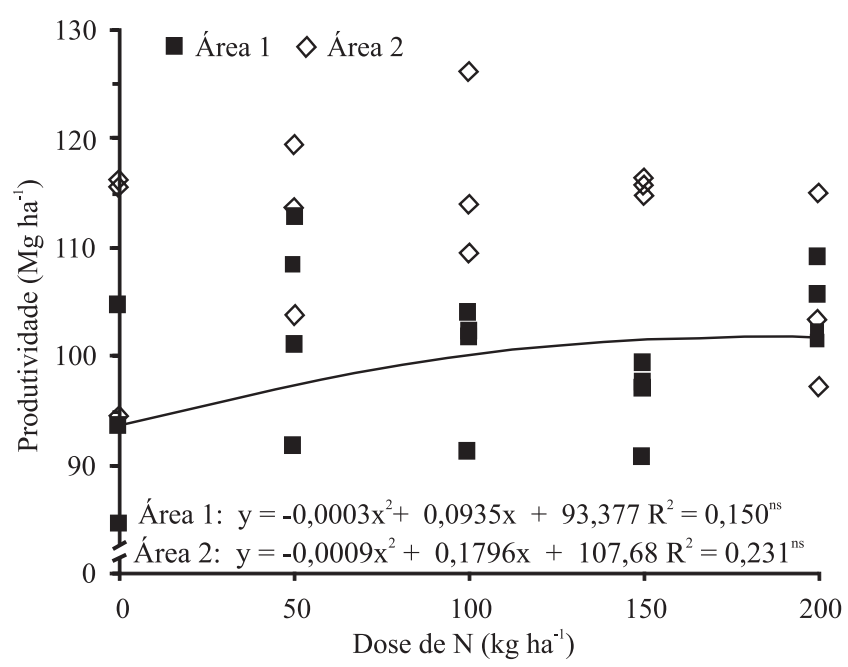

Figura 2. Produtividade final de colmos obtida em cada parcela das duas áreas experimentais, com equações de regressão não significativas.

Tabela 2. Contrastes ortogonais entre produtividade final de colmos nos tratamentos com e sem aplicação de $\mathrm{N}$ (testemunha).

\begin{tabular}{lcc}
\hline Contraste/tratamentos comparados & \multicolumn{2}{c}{ Produtividade de colmos $\left(\mathrm{Mg} \mathrm{ha}^{-1}\right)$} \\
\cline { 2 - 3 } & Área 1 & Área 2 \\
\hline $0 \mathrm{~kg} \mathrm{ha}^{-1}$ de N & $90,4^{*}$ & $108,7^{\text {ns }}$ \\
Demais doses & 101,0 & 112,4 \\
\hline
\end{tabular}

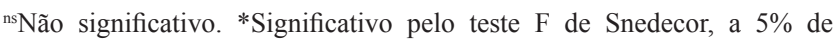
probabilidade. 
correlação significativa apenas na segunda avaliação (Tabela 5). Portanto, são necessárias mais pesquisas sobre a folha, a posição e o número de mensurações necessárias para a correta avaliação do teor de clorofila, por meio de clorofilômetro em cana-de-açúcar, o que pode explicar as baixas correlações obtidas, inclusive em comparação aos valores mensurados pelo sensor óptico. Argenta et al. (2001), ao avaliar características das plantas de milho como indicadores do nível de $\mathrm{N}$, constataram que a mensuração com o clorofilômetro foi o melhor indicador do nível de $\mathrm{N}$ na planta.

$\mathrm{O}$ sensor óptico diferenciou as doses de $\mathrm{N}$ apenas a partir da segunda avaliação (Tabela 4), em razão da pouca biomassa e da significativa interferência da cobertura morta do solo no campo de visão do equipamento na primeira avaliação (Shanahan et al., 2001) (Figura 3). Uma possível explicação é que, quando a distância do sensor ao alvo (dossel das plantas) está em torno de $1,0 \mathrm{~m}$ (recomendado pelo fabricante), o campo de visão do sensor é de cerca de $0,57 \mathrm{~m}$. Assim, com pouca biomassa existente, o sensor mensura predominantemente a reflectância do substrato palha e solo, o que interfere nos resultados.

A capacidade de os sensores ópticos diferenciarem as doses de $\mathrm{N}$ aplicadas também foi constatada por Povh et al. (2008) em cereais, Solari et al. (2008) em milho, e Inamasu et al. (2006) e Frasson (2007) em trabalhos preliminares com cana-de-açúcar.

Ao se compararem os diferentes índices de vegetação (NDVI e $\mathrm{CI}_{\text {âmbar }}$ ), verifica-se magnitude semelhante entre os valores obtidos (Tabelas 4 e 5), o que já era esperado por serem calculados a partir da reflectância dos mesmos comprimentos de onda. Assim, buscou-se comparar a sensibilidade dos índices de vegetação ao teor de clorofila (Figura 4). Com base em índice de suficiência (SI) de $95 \%$ para os valores SPAD (teor de clorofila) (Blackmer \& Schepers, 1995; Hurtado et al., 2011), observou-se que os SIs correspondentes são 92,5 e $87,5 \%$ para NDVI e $\mathrm{CI}_{\text {âmbar }}$ respectivamente. Isso indica maior sensibilidade do $\mathrm{CI}_{\text {âmbar }}$ em estimar a nutrição das plantas por N, o que corrobora Gitelson et al. (2003) e Solari et al. (2008). Esse comportamento pode ser atribuído à relação não linear existente entre o NDVI e o teor de clorofila da cultura, pois ocorre saturação do NDVI em altas concentrações de biomassa, mas não do $\mathrm{CI}_{\text {âmbar }}$ (Solari et al., 2008). No entanto, segundo Eitel et al. (2008), é difícil se obter um índice de vegetação que seja sensível ao teor de clorofila e insensível à quantidade de biomassa e à cobertura do solo. Portanto, são necessários mais estudos para comprovar essa inferência preliminar de maior sensibilidade do $\mathrm{CI}_{\text {âmbar }}$, em comparação ao NDVI.

Houve alta correlação entre as mensurações com o sensor óptico, na terceira avaliação (cultura com 0,6 m de altura), e a produtividade (Tabela 5). Nesse período, procedeu-se à coleta de dados com o sensor óptico, em área total. Para comparar as duas metodologias de recomendação de $\mathrm{N}$ em taxa variável (Met-CDR e Met-FRN), utilizou-se a resposta da cana ao N, mensurada pelo sensor óptico nos experimentos em parcelas, e a correlação entre a produtividade e os valores do índice, mensurados pelo sensor óptico (Figura 5), ambas na terceira avaliação.

Uma vez que os IVs oriundos do sensor óptico obtiveram resposta linear ao $\mathrm{N}$ aplicado (Tabela 4 e Figura 5), optou-se por determinar a dose de $\mathrm{N}$ máxima recomendada pela Met-CDR em função da extração e da exportação pela colheita. Para tanto, atribuiuse $1 \mathrm{~kg}$ de $\mathrm{N}$ para cada tonelada de cana colhida na safra anterior (Vitti \& Mazza, 2002; Cantarella et al., 2007), e o $\mathrm{N}$ máximo recomendado foi igual a 142 e

Tabela 3. Parâmetros de qualidade da cana-de-açúcar em função das doses de N utilizadas, nas duas áreas avaliadas.

\begin{tabular}{|c|c|c|c|c|c|c|c|c|}
\hline \multirow[t]{2}{*}{ Dose de N $\left(\mathrm{kg} \mathrm{ha}^{-1}\right)$} & \multicolumn{4}{|c|}{ Área 1} & \multicolumn{4}{|c|}{ Área 2} \\
\hline & Brix $(\%)$ & Pol (\%) & Fibra (\%) & $\operatorname{ATR}\left(\mathrm{kg} \mathrm{Mg}^{-1}\right)$ & Brix $(\%)$ & Pol $(\%)$ & Fibra (\%) & $\operatorname{ATR}\left(\mathrm{kg} \mathrm{Mg}^{-1}\right)$ \\
\hline 0 & 19,30 & 16,94 & 11,26 & 143,32 & 19,74 & 17,68 & 11,70 & 147,73 \\
\hline 50 & 19,71 & 17,44 & 11,05 & 147,73 & 19,35 & 17,29 & 11,53 & 145,08 \\
\hline 100 & 19,72 & 17,46 & 11,01 & 147,94 & 19,54 & 17,49 & 11,18 & 147,53 \\
\hline 150 & 19,45 & 17,14 & 11,08 & 145,29 & 19,55 & 17,46 & 11,44 & 146,67 \\
\hline 200 & 19,42 & 17,15 & 10,62 & 146,49 & 19,58 & 17,57 & 11,24 & 147,95 \\
\hline $\operatorname{Doses}^{(1)}$ & $0,355^{\mathrm{ns}}$ & $0,308^{\mathrm{ns}}$ & $0,052^{\mathrm{ns}}$ & $0,284^{\mathrm{ns}}$ & $0,838^{\mathrm{ns}}$ & $0,873^{\text {ns }}$ & $0,106^{\mathrm{ns}}$ & $0,833^{\text {ns }}$ \\
\hline $\mathrm{Blocos}^{(1)}$ & $0,085^{\mathrm{ns}}$ & $0,043^{*}$ & $0,219^{\mathrm{ns}}$ & $0,117^{\mathrm{ns}}$ & $0,866^{\mathrm{ns}}$ & $0,864^{\mathrm{ns}}$ & $0,001 * *$ & $0,547^{\mathrm{ns}}$ \\
\hline CV (\%) & 1,74 & 2,18 & 2,32 & 2,19 & 2,41 & 2,95 & 2,41 & 2,66 \\
\hline
\end{tabular}

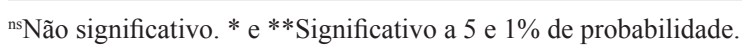


$125 \mathrm{~kg} \mathrm{ha}^{-1}$, respectivamente, para as áreas $1 \mathrm{e} 2$. Desse modo, um valor qualquer do índice de vegetação indicaria quantidade teórica de $\mathrm{N}$ disponibilizado e absorvido pelo solo. Recomenda-se a diferença entre

Tabela 4. Índice de vegetação por diferença normalizada (NDVI) e índice de clorofila ( $\left.\mathrm{CI}_{\text {âmbar }}\right)$ obtidos com sensor óptico, teor foliar de clorofila obtido com clorofilômetro (SPAD) e teor foliar de $\mathrm{N}\left(\mathrm{g} \mathrm{kg}^{-1}\right)$, nas três avaliações realizadas em canade-açúcar, nas duas áreas experimentais, em função das doses de $\mathrm{N}$ aplicadas, com seus respectivos efeitos de tratamentos (doses), blocos e significância de modelos de regressão linear e quadrático.

\begin{tabular}{|c|c|c|c|c|c|c|c|c|}
\hline \multirow{2}{*}{$\begin{array}{l}\text { Dose de N } \\
\left(\mathrm{kg} \mathrm{ha}^{-1}\right)\end{array}$} & \multicolumn{4}{|c|}{ Área 1} & \multicolumn{4}{|c|}{ Área 2} \\
\hline & NDVI & $\mathrm{CI}_{\text {âmbar }}$ & SPAD & $\mathrm{N}$ foliar & NDVI & $\mathrm{CI}_{\text {âmbar }}$ & SPAD & $\mathrm{N}$ foliar \\
\hline & \multicolumn{8}{|c|}{ Primeira avaliação - colmos com altura média de $0,2 \mathrm{~m}$} \\
\hline 0 & 0,338 & 1,09 & 46,08 & 20,88 & 0,339 & 1,17 & 42,93 & 20,89 \\
\hline 50 & 0,338 & 1,07 & 45,98 & 21,46 & 0,358 & 1,23 & 42,95 & 19,73 \\
\hline 100 & 0,336 & 1,07 & 46,98 & 21,97 & 0,360 & 1,24 & 43,73 & 19,41 \\
\hline 150 & 0,340 & 1,09 & 45,90 & 20,48 & 0,360 & 1,24 & 42,98 & 25,13 \\
\hline 200 & 0,338 & 1,07 & 46,23 & 21,78 & 0,366 & 1,25 & 43,78 & 19,72 \\
\hline Blocos $^{(1)}$ & $0,002 *$ & $0,005^{*}$ & $0,104^{\mathrm{ns}}$ & $0,399^{\text {ns }}$ & $0,202^{\mathrm{ns}}$ & $0,049 *$ & $0,045^{*}$ & $0,539^{\text {ns }}$ \\
\hline $\operatorname{Doses}^{(1)}$ & $0,983^{\mathrm{ns}}$ & $0,940^{\mathrm{ns}}$ & $0,797^{\mathrm{ns}}$ & $0,466^{\mathrm{ns}}$ & $0,235^{\mathrm{ns}}$ & $0,420^{\mathrm{ns}}$ & $0,709^{\mathrm{ns}}$ & $0,066^{\mathrm{ns}}$ \\
\hline Regressão linear ${ }^{(1)}$ & - & - & - & - & - & - & - & - \\
\hline Regressão quadrática ${ }^{(1)}$ & - & - & - & - & - & - & - & - \\
\hline \multirow[t]{2}{*}{$\mathrm{CV}(\%)$} & 2,50 & 3,66 & 2,93 & 6,01 & 4,54 & 4,76 & 2,76 & 13,31 \\
\hline & \multicolumn{8}{|c|}{ Segunda avaliação - colmos com altura média de $0,4 \mathrm{~m}$} \\
\hline 0 & 0,489 & 1,98 & 44,25 & 20,79 & 0,451 & 1,73 & 43,30 & 21,01 \\
\hline 50 & 0,525 & 2,27 & 47,13 & 23,58 & 0,491 & 2,03 & 45,38 & 22,41 \\
\hline 100 & 0,523 & 2,25 & 48,08 & 23,93 & 0,507 & 2,19 & 45,58 & 25,54 \\
\hline 150 & 0,525 & 2,28 & 47,93 & 25,35 & 0,522 & 2,29 & 46,48 & 24,49 \\
\hline 200 & 0,538 & 2,39 & 47,93 & 24,64 & 0,523 & 2,30 & 47,15 & 26,69 \\
\hline Blocos $^{(1)}$ & $0,024 *$ & $0,018^{*}$ & $0,001^{\mathrm{ns}}$ & $0,370^{\mathrm{ns}}$ & $0,169^{\text {ns }}$ & $0,146^{\mathrm{ns}}$ & $0,206^{\mathrm{ns}}$ & $0,488^{\mathrm{ns}}$ \\
\hline $\operatorname{Doses}^{(1)}$ & $0,003 * *$ & $0,002 * *$ & $0,003 * *$ & $0,051^{\mathrm{ns}}$ & $0,013 *$ & $0,018^{*}$ & $0,003^{*}$ & $0,015^{*}$ \\
\hline Regressão linear ${ }^{(1)}$ & $0,001 * *$ & $0,001 * *$ & $0,001 * *$ & - & $0,001 * *$ & $0,002 * *$ & $0,001 * *$ & $0,002 * *$ \\
\hline Regressão quadrática & $0,116^{\mathrm{ns}}$ & $0,132^{\mathrm{ns}}$ & $0,010 *$ & - & $0,146^{\mathrm{ns}}$ & $0,145^{\mathrm{ns}}$ & $0,321^{\mathrm{ns}}$ & $0,524^{\mathrm{ns}}$ \\
\hline \multirow[t]{2}{*}{ CV $(\%)$} & 2,59 & 4,71 & 2,55 & 8,20 & 5,33 & 10,63 & 2,38 & 8,77 \\
\hline & \multicolumn{8}{|c|}{ Terceira avaliação - colmos com altura média de $0,6 \mathrm{~m}$} \\
\hline 0 & 0,522 & 2,22 & 44,10 & 20,29 & 0,516 & 2,23 & 45,85 & 20,03 \\
\hline 50 & 0,548 & 2,47 & 45,45 & 20,43 & 0,555 & 2,57 & 46,30 & 20,53 \\
\hline 100 & 0,552 & 2,52 & 45,78 & 19,12 & 0,550 & 2,56 & 47,45 & 21,51 \\
\hline 150 & 0,552 & 2,52 & 46,18 & 20,33 & 0,578 & 2,80 & 47,18 & 20,44 \\
\hline 200 & 0,568 & 2,69 & 47,48 & 19,70 & 0,580 & 2,83 & 47,25 & 21,90 \\
\hline $\mathrm{Blocos}^{(1)}$ & $0,005 * *$ & $0,008 * *$ & $0,011 *$ & $0,063^{\mathrm{ns}}$ & $0,133^{\mathrm{ns}}$ & $0,123^{\mathrm{ns}}$ & $0,060^{\mathrm{ns}}$ & $0,069^{\mathrm{ns}}$ \\
\hline $\operatorname{Doses}^{(1)}$ & $0,001 * *$ & $0,001 * *$ & $0,007 * *$ & $0,388^{\mathrm{ns}}$ & $0,010 * *$ & $0,009 * *$ & $0,297^{\mathrm{ns}}$ & $0,241^{\mathrm{ns}}$ \\
\hline Regressão linear ${ }^{(1)}$ & $0,001 * *$ & $0,001 * *$ & $0,001 * *$ & - & $0,001 * *$ & $0,001 * *$ & - & - \\
\hline Regressão quadrática ${ }^{(1)}$ & $0,115^{\mathrm{ns}}$ & $0,196^{\mathrm{ns}}$ & $0,989^{\text {ns }}$ & - & $0,348^{\mathrm{ns}}$ & $0,361^{\mathrm{ns}}$ & - & - \\
\hline CV (\%) & 1,39 & 3,28 & 2,17 & 5,25 & 4,03 & 7,86 & 2,52 & 5,99 \\
\hline
\end{tabular}

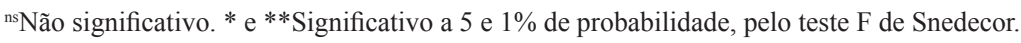

Tabela 5. Coeficientes de correlação linear entre os valores de índices de vegetação da cana-de-açúcar, obtidos com sensor óptico, e as variáveis avaliadas, nas áreas 1 e 2, na segunda avaliação, com colmo com 0,4 m de altura (acima da diagonal), e, na terceira avaliação, com colmo com $0,6 \mathrm{~m}$ de altura (abaixo da diagonal).

\begin{tabular}{|c|c|c|c|c|c|}
\hline Variável & NDVI & CIâmbar & Clorofilômetro & Teor de $\mathrm{N}$ foliar & Produtividade \\
\hline NDVI & - & $0,992 * *$ & $0,936 * *$ & $0,757 * *$ & $0,436^{\mathrm{ns}}$ \\
\hline CIâmbar & $0,993 * *$ & - & $0,917 * *$ & $0,816 * *$ & $0,527^{\mathrm{ns}}$ \\
\hline Clorofilômetro & $0,750 * *$ & $0,809 * *$ & - & $0,732 * *$ & $0,319^{\text {ns }}$ \\
\hline Teor de $\mathrm{N}$ foliar & $0,305^{\mathrm{ns}}$ & $0,359^{\mathrm{ns}}$ & $0,382^{\mathrm{ns}}$ & - & $0,667^{*}$ \\
\hline Produtividade & $0,837 * *$ & $0,880 * *$ & $0,820 * *$ & $0,513^{\mathrm{ns}}$ & - \\
\hline
\end{tabular}

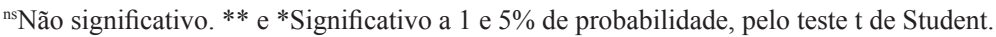


a dose máxima de $\mathrm{N}$, para cada área experimental, e a quantidade de $\mathrm{N}$ absorvida (Figura 5).

Inicialmente, a Met-FRN requer a estimativa da produtividade a ser obtida na safra. No presente trabalho, utilizou-se a equação oriunda da correlação linear entre a produtividade de colmos e os IVs obtidos nos experimentos em parcelas. Foram obtidos coeficientes de determinação $\left(\mathrm{R}^{2}\right)$ de 0,701 e 0,775 , para o NDVI e o $\mathrm{CI}_{\text {âmbar, }}$ respectivamente. Assim, cada ponto registrado pelo sensor óptico teve seu potencial produtivo atual estimado (YP0, Equações 4 e 5), bem como o índice de resposta, o potencial produtivo almejado com a aplicação de $\mathrm{N}$ (YPN) e a dose de $\mathrm{N} \mathrm{a}$ ser aplicada (Equação 3).

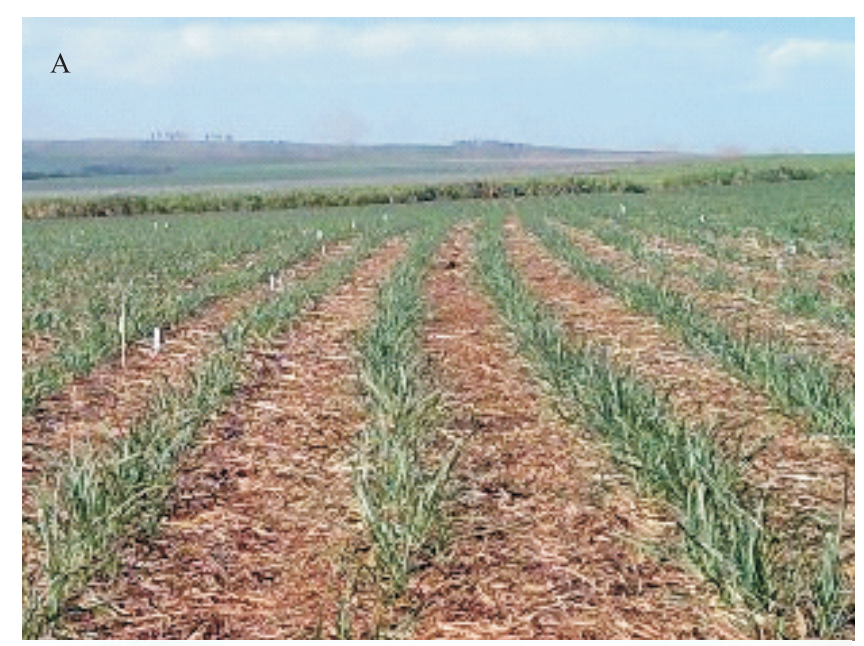

B

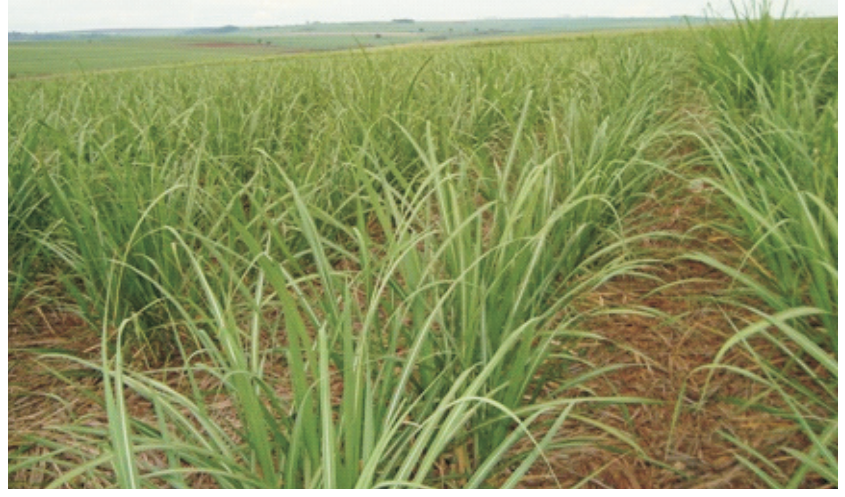

Figura 3. Cobertura do solo pela cultura da cana de açúcar no momento da primeira (colmo com 0,2 m de altura) (A) e da segunda (colmo com 0,4 m de altura) (B) avaliação.
YP0 $=296,1112735$ NDVI - 60,4881506

$\mathrm{YP} 0=31,7312106 \mathrm{CI}_{\text {âmbar }}+22,3738808$

Ao se analisar as estratégias de recomendação, inferese que a Met-CDR tem como objetivo principal atingir a máxima produção, com a aplicação da quantidade necessária de fertilizante, enquanto a Met-FRN busca economizar fertilizante, ao aplicar mais $\mathrm{N}$ apenas onde a cultura apresenta maior potencial produtivo. Logo, na primeira estratégia, quanto menor o valor do índice de vegetação mensurado, maior será a dose de $\mathrm{N}$ recomendada. No entanto, na segunda, quanto menor o valor do índice de vegetação, menor a produtividade esperada, e, consequentemente, menor será a dose de fertilizante recomendada.

Verificou-se que, ao se adotar a Met-CDR, a maior parte dos talhões receberia doses superiores a $100 \mathrm{~kg} \mathrm{ha}^{-1}$ de $\mathrm{N}$, o que poderia inviabilizar economicamente a adoção dessa estratégia. Quanto à utilização da Met-FRN, a maior parte do talhão receberia doses inferiores a $40 \mathrm{~kg} \mathrm{ha}^{-1}$, que, se comprovadamente não limitassem a produção, seria um grande atrativo para a adoção dessa metodologia por parte dos produtores.

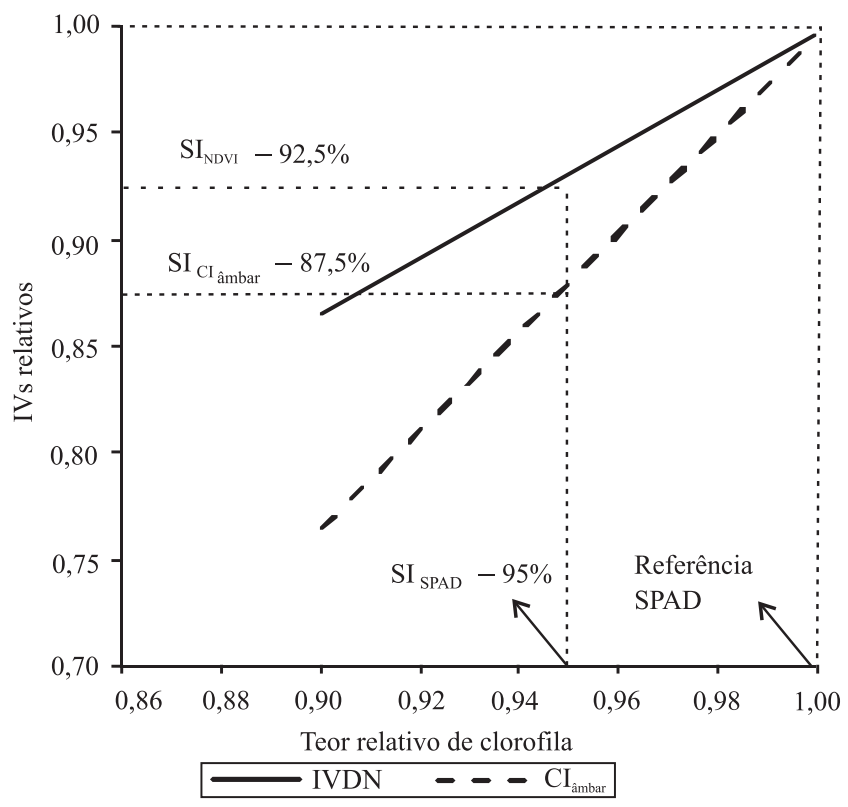

Figura 4. Relação linear entre o teor relativo de clorofila e os índices de vegetação (IV) (NDVI e $\mathrm{CI}_{\text {âmbar }}$ ) mensurados na segunda avaliação (colmo com 0,4 $\mathrm{m}$ de altura), índice de suficiência (SI) de 95\% das leituras realizadas com clorofilômetro e respectivos SIs dos índices de vegetação. 
A Met-CDR não leva em consideração a necessidade de se aplicar $\mathrm{N}$ de acordo com a capacidade de resposta da cultura durante a safra, para aumentar a eficiência de uso desse elemento, o que pode causar problemas de sub e superestimação, pela atuação de outros fatores limitantes, que não a disponibilidade de N. Assim, acredita-se que a Met-FRN tenha sido a metodologia mais adequada nas condições deste experimento, por recomendar doses baixas de $\mathrm{N}$ (Tabela 6), condizente
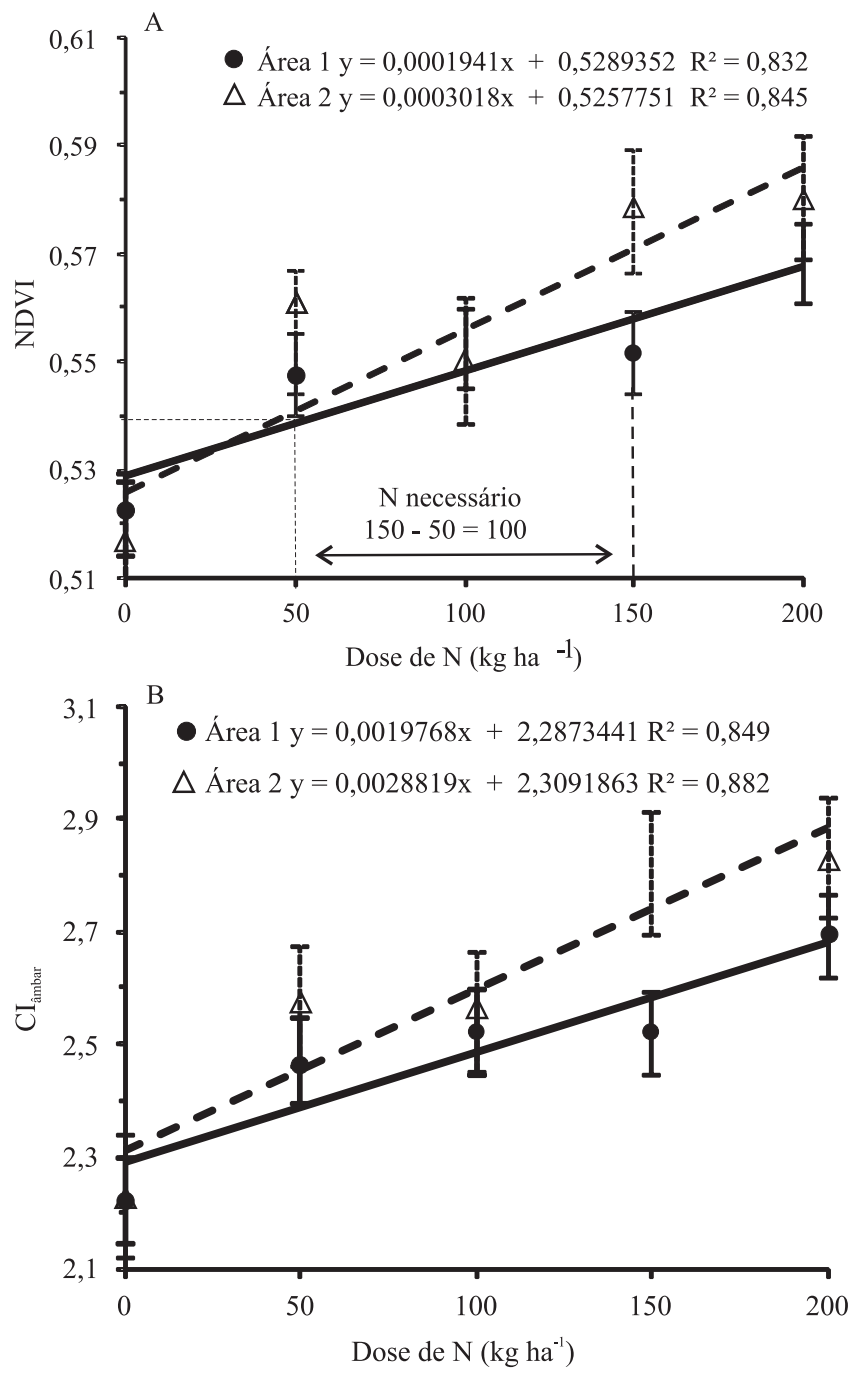

Figura 5. Curvas de dose/resposta dos valores médios de NDVI (A) e CI âmbar (B), mensurados com sensor ótico na terceira avaliação (colmo com $0,6 \mathrm{~m}$ de altura), dos experimentos em parcelas, suas respectivas equações para recomendação de $\mathrm{N}$ e exemplificação do cálculo para determinação do N necessário, em função do NDVI (A). As barras verticais indicam o erro padrão da média. com a resposta da cultura ao $\mathrm{N}$ verificada nos experimentos em parcelas.

Em relação aos diferentes índices de vegetação ( $\mathrm{CI}_{\text {âmbar }}$ e NDVI), observou-se que, ao se utilizar o $\mathrm{CI}_{\text {âmbar, }}$ a quantidade de $\mathrm{N}$ recomendada foi sempre mais elevada e com maior amplitude de variação entre as doses, o que indica maior sensibilidade desse índice para a cultura da cana-de-açúcar.

Os modelos de recomendação avaliados no presente trabalho foram obtidos a partir dos resultados de experimentos em parcelas alocadas em áreas supostamente homogêneas, o que limita, quando da extrapolação desses dados, o correto tratamento da variabilidade identificada em áreas comerciais. Para a implementação de um estudo dessa natureza em áreas extensas e com variabilidade intrínseca, é necessária a obtenção de mapas de produtividade com dados confiáveis, o que não está disponível no momento. Além disso, neste trabalho, apenas um ciclo agrícola foi considerado, e a resposta ao $\mathrm{N}$, em virtude de condições edafoclimáticas distintas, pode ter interferido nos resultados. Em função dessas limitações, outros ensaios em pequenas áreas são fundamentais.

São necessários mais estudos sobre as estratégias de recomendação de $\mathrm{N}$, com avaliação da resposta obtida por meio da produtividade ao longo dos anos, bem como dos índices de vegetação que melhor se adaptem

Tabela 6. Estatística descritiva da quantidade de nitrogênio recomendada $\left(\mathrm{kg} \mathrm{ha}^{-1}\right)$, por meio de diferentes metodologias de recomendação, nas duas áreas experimentais.

\begin{tabular}{lrrrrr}
\hline Estatística & \multicolumn{2}{c}{$\mathrm{CI}_{\text {âmbar }}$} & & \multicolumn{2}{c}{ NDVI } \\
\cline { 2 - 3 } \cline { 5 - 6 } & $\begin{array}{c}\text { Curva dose- } \\
\text { resposta }\end{array}$ & $\begin{array}{c}\text { Faixa rica } \\
\text { em N }\end{array}$ & $\begin{array}{c}\text { Curva dose- } \\
\text { resposta }\end{array}$ & $\begin{array}{c}\text { Faixa rica } \\
\text { em N }\end{array}$ \\
\hline Média & 124,0 & 89,2 & 120,8 & 34,5 \\
Mediana & 142,0 & 89,5 & 142,0 & 36,4 \\
Moda & 142,0 & 0,0 & 142,0 & 0,0 \\
Desvio padrão & 42,3 & 48,5 & 45,6 & 18,3 \\
Mínimo & 0,0 & 0,0 & 0,0 & 0,0 \\
Máximo & 142,0 & 233,8 & 142,0 & 67,4 \\
Soma & $390.302,4$ & $280.789,1$ & $380.167,1$ & $108.761,2$ \\
\hline \multicolumn{5}{c}{ Área 2} \\
Média & 103,9 & 81,1 & 95,7 & 31,1 \\
Mediana & 125,0 & 81,1 & 125,0 & 31,6 \\
Moda & 125,0 & 0,0 & 125,0 & 0,0 \\
Desvio padrão & 39,6 & 41,9 & 44,9 & 16,5 \\
Mínimo & 0,0 & 0,0 & 0,0 & 0,0 \\
Máximo & 125,0 & 206,6 & 125,0 & 69,3 \\
Soma & $708.983,1$ & $553.481,3$ & $653.179,4$ & $212.351,3$ \\
\hline
\end{tabular}


às características da cultura da cana-de-açúcar e dos métodos comparativos que validem as mensurações realizadas com sensores ópticos, em diferentes safras e situações de cultivo.

\section{Conclusão}

O sensor óptico testado é ferramenta útil para auxiliar na recomendação da adubação nitrogenada de cobertura na cultura da cana-de-açúcar, em condições de variabilidade espacial da demanda de $\mathrm{N}$, e recomendação com base na resposta da cultura, estimada em faixa de cana-de-açúcar que tenha recebido dose adequada desse elemento.

\section{Agradecimentos}

À Financiadora de Estudos e Projetos, projeto PROSENSAP, e ao Conselho Nacional de Desenvolvimento Científico e Tecnológico, pelo apoio financeiro; à Fundação de Amparo à Pesquisa do Estado de São Paulo, pela concessão de bolsa; e à Usina São Martinho, ao Centro de Tecnologia Canavieira e à Jacto Máquinas Agrícolas, pela parceria e pelo auxílio no planejamento, na instalação, na condução e na colheita dos experimentos.

\section{Referências}

ARGENTA, G.; SILVA, P.R.F. da; MIELNICZUK, J.; BORTOLINI, C.G. Parâmetros de planta como indicadores do nível de nitrogênio na cultura do milho. Pesquisa Agropecuária Brasileira, v.37, p.519-527, 2001.

BATAGLIA, O.C.; FURLANI, A.M.C.; TEIXEIRA, J.P.F.; FURLANI, P.R.; GALLO, J.R. Métodos de análise química de plantas. Campinas: Instituto Agronômico, 1983. 48p. (IAC. Boletim técnico, 78).

BLACKMER, T.M.; SCHEPERS, J.S. Use of chlorophyll meter to monitor nitrogen status and schedule fertigation for corn. Journal of Production Agriculture, v.8, p.56-60, 1995.

BLACKMER, T.M.; SCHEPERS, J.S.; VARVEL, G.E.; WALTER-SHEA, E.A. Nitrogen deficiency detection using reflected shortwave radiation from irrigated corn canopies. Agronomy Journal, v.88, p.1-5, 1996.

CANTARELLA, H.; TRIVELIN, P.C.O.; VITTI, A.C. Nitrogênio e enxofre na cultura da cana-de-açúcar. In: YAMADA, T.; ABDALLA, S.R.S.; VITTI, G.C. (Ed.). Nitrogênio e enxofre na agricultura brasileira. Piracicaba: IPNI Brasil, 2007. p.355-412.

EITEL, J.U.H.; LONG, D.S.; GESSLER, P.E.; HUNT, E.R. Combined spectral index to improve ground-based estimates of nitrogen status in dryland wheat. Agronomy Journal, v.100, p.1694-1702, 2008.
FERREIRA, D.F. Sisvar: um programa para análises e ensino de estatística. Revista Symposium, v.6, p.36-41, 2008.

FRASSON, F.R. Utilização de sensor ótico ativo em cana-de-açúcar. 2007. 76p. Dissertação (Mestrado) - Escola Superior de Agricultura Luiz de Queiroz, Piracicaba.

GITELSON, A.A.; VIÑA, A.; ARKEBAUER, T.J.; RUNDQUIST, D.C.; KEYDAN, G.; LEAVITT, B. Remote estimation of leaf area index and green leaf biomass in maize canopies. Geophysical Research Letter, v.30, p.1248, 2003.

GROHS, D.S.; BREDEMEIER, C.; MUNDSTOCK, C.M.; POLETTO, N. Modelo para estimativa do potencial produtivo em trigo e cevada por meio do sensor GreenSeeker. Engenharia Agrícola, v.29, p.101-112, 2009.

HURTADO, S.M.C.; RESENDE, A.V. de; SILVA, C.A. da; CORAZZA, E.J.; SHIRATSUCHI, L.S. Clorofilômetro no ajuste da adubação nitrogenada em cobertura para o milho de alta produtividade. Ciência Rural, v.41, p.1011-1017, 2011.

HURTADO, S.M.C.; RESENDE, A.V. de; SILVA, C.A. da; CORAZZA, E.J.; SHIRATSUCHI, L.S. Variação espacial da resposta do milho à adubação nitrogenada de cobertura em lavoura no cerrado. Pesquisa Agropecuária Brasileira, v.44, p.300-309, 2009.

INAMASU, R.Y.; SOUSA, R.V.; PORTO, A.J.V.; FORTES, C.; LUCHIARI, A.; SCHEPERS, J.S.; SHANAHAN, J.F.; FRANCIS, D.D. Acesso ao estado nutricional da cana-de-açúcar por meio de sensor ativo de refletância. In: CONGRESSO BRASILEIRO DE AGRICUlturA DE PRECISÃO, 2., 2006, São Pedro. Anais. Piracicaba: ESALQ, 2006. CD-ROM.

JARRELL, W.M.; BEVERLY, R.B. The dilution effect in plant nutrition studies. Advances in Agronomy, v.34, p.197-224, 1981.

LUKINA, E.V.; FREEMAN, K.W.; WYNN, K.J.; THOMASON, W.E.; MULLEN, R.W.; STONE, M.L.; SOLIE, J.B.; KLATT, A.R.; JOHNSON, G.V.; ELLIOTT, R.L.; RAUN, W.R. Nitrogen fertilization optimization algorithm based on in-season estimates of yield and plant nitrogen uptake. Journal of Plant Nutrition, v.24, p.885-898, 2001.

MOTOMIYA, A.V. de A.; MOLIN, J.P.; CHIAVEGATO, E.J. Utilização de sensor óptico ativo para detectar deficiência foliar de nitrogênio em algodoeiro. Revista Brasileira de Engenharia Agrícola e Ambiental, v.13, p.137-145, 2009.

POVH, F.P. Utilização de sensor ótico ativo em culturas de cereais. 2007. 86p. Dissertação (Mestrado) - Escola Superior de Agricultura Luiz de Queiroz, Piracicaba.

POVH, F.P.; MOLIN, J.P.; GIMENEZ, L.M.; PAULETTI, V.; MOLIN, R.; SALVI, J.V. Comportamento do NDVI obtido por sensor ótico ativo em cereais. Pesquisa Agropecuária Brasileira, v.43, p.1075-1083, 2008.

PRADO, H. Ambientes de produção de cana-de-açúcar na região Centro-Sul do Brasil. Informações Agronômicas, v.110, p.12-17, 2005. Encarte técnico.

RAIJ, B. van; ANDRADE, J.C. de; CANTARELLA, H.; QUAGGIO, J.A. (Ed.). Análise química para avaliação da fertilidade de solos tropicais. Campinas: IAC, 2001. 285p. 
RAIJ, B. van; CANTARELLA, H. Outras culturas industriais. In: RAIJ, B. van; CANTARELLA, H.; QUAGGIO, J.A.; FURLANI, A.M.C. (Ed.). Recomendações de adubação e calagem para o Estado de São Paulo. 2.ed. Campinas: IAC, 1997. p.189-203. (IAC. Boletim técnico, 100).

RAMBO, L.; SILVA, P.R.F. da; STRIEDER, M.L.; SILVA, A.A. da; SANGOI, L.; VIEIRA, V.M. Índices nutricionais de $\mathrm{N}$ e produtividade de milho em diferentes níveis de manejo e de adubação nitrogenada. Pesquisa Agropecuária Brasileira, v.46, p.390-397, 2011.

RAUN, W.R.; SOLIE, J.B.; JOHNSON, G.V.; STONE, M.L.; MULLEN, R.W.; FREEMAN, K.W.; THOMASON, W.E.; LUKINA, E.V. Improving nitrogen use efficiency in cereal grain production with optical sensing and variable rate application. Agronomy Journal, v.94, p.815-820, 2002.

SEGATO, S.V.; PINTO, A. de S.; JENDIROBA, E.; NÓBREGA, J.C.M. de. Atualização em produção em cana-de-açúcar. Piracicaba: Livroceres, 2006. 415p.

SHANAHAN, J.F.; SCHEPERS, J.S.; FRANCIS, D.D.; VARVEL, G.E.; WILHELM, W.W.; TRINGE, J.M.; SCHLEMMER, M.S.; MAJOR, D.J. Use of remote sensing imagery to estimate corn grain yield. Agronomy Journal, v.93, p.583-589, 2001.
SOLARI, F. Developing a crop based strategy for on-the-go nitrogen management in irrigated cornfields. 2006. 157p. Thesis $(\mathrm{PhD})$ - University of Nebraska, Lincoln.

SOLARI, F.; SHANAHAN, J.; FERGUSON, R.B.; SCHEPERS, J.S.; GITELSON, A.A. Active sensor reflectance measurements of corn nitrogen status and yield potential. Agronomy Journal, v.100, p.571-579, 2008.

SUI, R.; WILKERSON, J.B.; HART, W.E.; WILHELM, L.R.; HOWARD, D.D. Multi-spectral sensor for detection of nitrogen status in cotton. Applied Engineering in Agriculture, v.21, p.167-172, 2005.

TEAL, R.K.; TUBANA, B.; GIRMA, K.; FREEMAN, K.W.; ARNALL, D.B.; WALSH, O.; RAUN, R.W. In-season prediction of corn grain yield potential using normalized difference vegetation index. Agronomy Journal, v.98, p.1488-1494, 2006.

VITTI, A.C.; TRIVELIN, P.C.O.; GAVA, G.J.G.; PENATTI, C.P.; BOLOGNA, I.R.; FARONI, C.E.; FRANCO, H.C.J. Produtividade da cana-de-açúcar relacionada ao nitrogênio residual da adubação e do sistema radicular. Pesquisa Agropecuária Brasileira, v.42, p.249-256, 2007.

VITTI, G.C.; MAZZA, J.A. Planejamento, estratégias de manejo e nutrição da cultura de cana-de-açúcar. Piracicaba: POTAFOS, 2002. 16p. Encarte técnico.

Recebido em 25 de novembro de 2010 e aprovado em 21 de novembro de 2011 